Textures and Microstructures, Vol. 33, pp. 291-301 Reprints available directly from the publisher Photocopying permitted by license only
(C) 1999 OPA (Overseas Publishers Association) N.V.

Published by license under the Gordon and Breach Science Publishers imprint. Printed in Malaysia.

\title{
RESIDUAL STRESS AND TEXTURE DUE TO COLD AND HOT EXTRUSION PROCESSES
}

\author{
A. PYZALLA* and W. REIMERS \\ Hahn-Meitner-Institut, Strukturforschung, Glienicker Straße 100, \\ D-14109 Berlin, Germany
}

\begin{abstract}
The residual stress state and the texture of cold forward extruded full and hollow steel bodies as well as a hot extruded $\mathrm{AlSi} 25 \mathrm{Cu} 4 \mathrm{Mg}$ l tube are studied by $\mathrm{X}$-ray, high energy synchrotron and neutron diffraction. The experimental results reveal that all samples are fibre textured and that there are characteristic distributions of the residual stresses vs. sample diameter. In case of the cold forward extruded samples at low degrees of natural strain, the rod kernel is under compressive residual stresses which are balanced by tensile residual stresses in the outer part of the sample. In contrast to this, the outer part of the hot extruded sample is under compressive macroscopic stresses which are balanced by tensile macroscopic residual stresses in the inner part of the sample.
\end{abstract}

Keywords: Residual stresses; Neutrons; Extrusion; Texture; Synchrotron

\section{INTRODUCTION}

The strong plastic deformation and the macroscopic deformation gradient during the extrusion process lead to the formation of grain deformation, texture and residual stresses. The microstructure, the texture and especially the residual stress state established during an extrusion process are of great practical interest regarding the static and dynamic mechanical properties of the material as well as the workpiece's resistance to shock and fatigue failure. Therefore, the texture and the residual stress state of cold forward extruded steel samples and

*Corresponding author. E-mail: pyzalla@hmi.de. 
the hot forward extruded AlSi-alloy AlSi25CU4Mg1 are investigated by $\mathrm{X}$-ray, neutron and high energy synchrotron diffraction. The use of non-destructive methods is most profitable here, since considerably differing results have been reported after the determination of the residual stresses in extruded samples by destructive methods (Frisch and Thomsen, 1957; Moore and Evans, 1958), while residual stress analysis by neutron diffraction (Modlen et al., 1992; Genzel et al., 1996) revealed systematic relations between process parameters and the resulting residual stress state of the specimens. Furthermore, in case of the hot forward extruded multiphase AlSi25Cu4Mg1-alloy, phase specific residual microstresses, which only can be determined by diffraction methods, arise due to the deformation process as well as due to cooling.

\section{EXPERIMENTAL SET-UP}

\section{Samples}

Full and hollow samples, German steel grade C15, were cold forward extruded (Figs. 1 and 2) at the Institute for Metal Forming, University

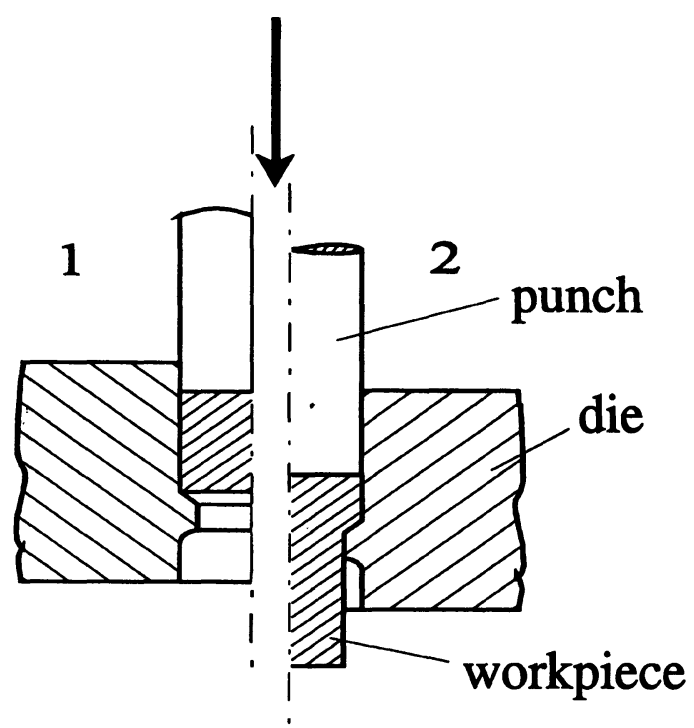

FIGURE 1 Principle of full forward extrusion. 


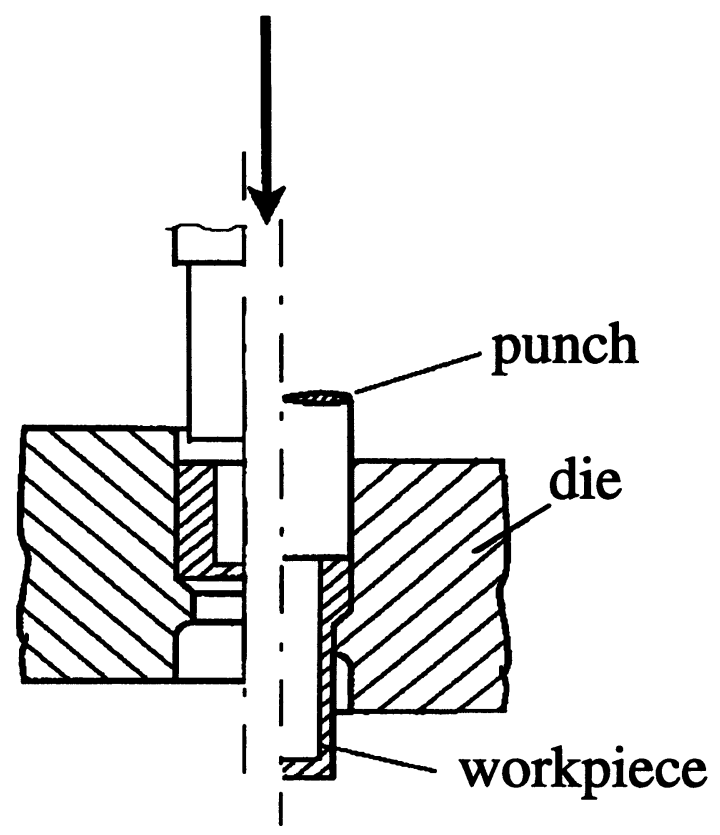

FIGURE 2 Principle of hollow forward extrusion.

of Stuttgart, Germany. The degree of natural strain $\varphi$ was varied between $\varphi=0.5,1.2$ and 1.6 in case of full forward extruded samples and between $\varphi=0.5$ and 1.2 in case of the hollow samples. The AlSi25$\mathrm{Cu} 4 \mathrm{Mg} 1$ tubes were hot forward extruded at the "Forschungszentrum Strangpressen" of the Technical University in Berlin, Germany. The extrusion process was performed indirectly using a moving mandrel. The temperature of the workpiece was $360^{\circ} \mathrm{C}$ app., the degree of natural strain is $\varphi=2.8$.

\section{Texture Characterisation and Residual Stress Analysis}

For texture analysis an X-ray diffractometer with an Eulerian cradle was employed. In case of the steel samples the reflections 110, 211 and 200 were chosen for texture characterisation. In case of the hot extruded aluminium alloy 111,200 and 220 pole figures were measured.

The X-ray residual stress analysis on the steel samples was performed by the $\sin ^{2} \psi$-method (Macherauch and Müller, 1961) on a 
$\psi$-diffractometer using $\mathrm{Cr}-\mathrm{K} \alpha$-radiation and investigating the 200 and 211 reflections. In order to obtain a residual stress profile, part of the sample was etched electrochemically and measurements were carried out after that.

For neutron diffraction the instrument E3 of the BERII reactor at the Hahn-Meitner-Institut, Berlin, Germany was used. A wavelength $\lambda=0.13880 \mathrm{~nm}$ (Cu-monochromator, 220) was chosen. In case of the steel samples this covers the reflections $110 / 220,211$ and 200 within the experimentally accessible range $2 \theta \approx 40-120^{\circ}$ of the multichannel counter used for radiation detection. The diffracting volume was limited by conical cadmium slits to $2 \times 2 \times 2 \mathrm{~mm}^{3}$. In order to account for the texture and the plastic anisotropy, the difference between the axial and the radial residual stress component was evaluated from the slope of the $d$ vs. $\sin ^{2} \psi$ curves. Whereas the $d$ vs. $\sin ^{2} \psi$ curves were linear in case of the hollow samples, non-linear $d$ vs. $\sin ^{2} \psi$ curves were obtained in the rod kernel of the full forward extruded sample and thus were evaluated using appropriate methods (Hauk and Sesemann, 1976; 1985; Hauk et al., 1990). For the interplanar lattice spacing $d_{0}$ of the $\mathrm{Si}$ particles in the AlSi25Cu4Mg1, the $d$-value of pure Si according to the JCPDS was used. The $d_{0}$ of the Al-alloy matrix was calculated from equilibrium conditions.

Residual stress analysis using high energy synchrotron radiation was performed at the beam line ID 15A of the ESRF Grenoble. Due to the high photon flux and the parallel beam the volume element in this case could be restricted to a small parallelepiped of $1.65 \mathrm{~mm} \times 0.145 \mathrm{~mm}$. The $d_{0}$ value necessary for the determination of the three-dimensional residual stress state was calculated as an average of the $d$-values obtained for the different reflections and volume elements ('randomwalk-method').

\section{RESULTS AND DISCUSSION}

\section{Cold Forward Extrusion}

The microstructure of a cold forward extruded steel sample reveals severe grain elongation caused by the plastic deformation during 
the extrusion process (Fig. 3). The plastic deformation also leads to the development of a $\langle 110\rangle$-fibre texture (Fig. 4), characteristic for bcc steels (Wassermann and Grewen, 1962). This fibre texture is more pronounced in the rod kernel than in the outer part of the samples.

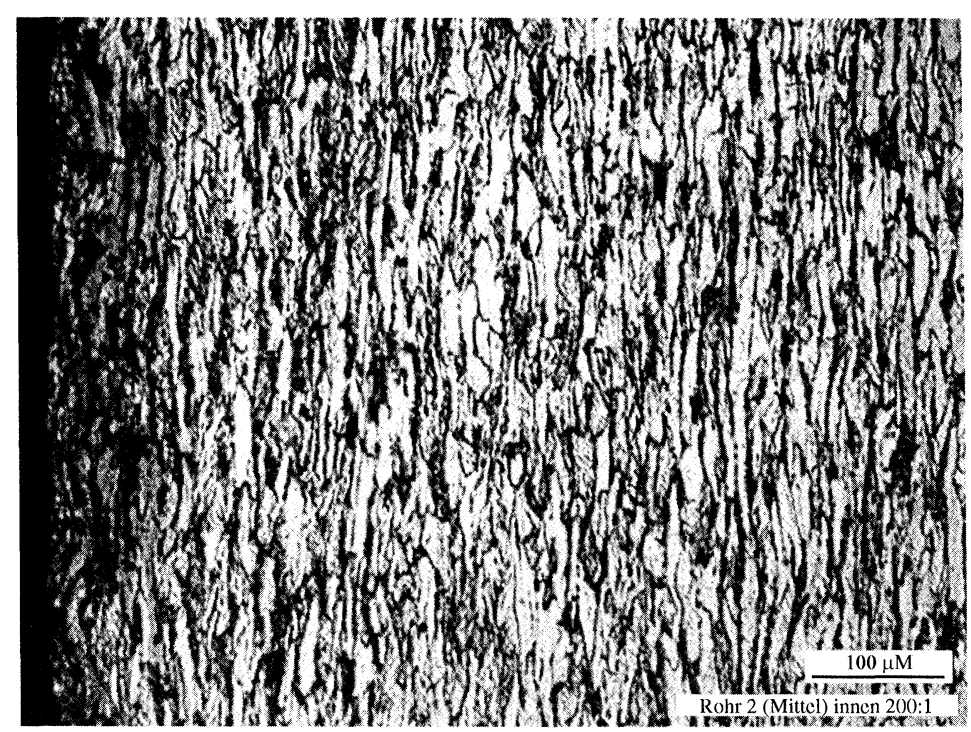

FIGURE 3 Microstructure in the rod kernel, $\varphi=1.2$, steel grade C15.

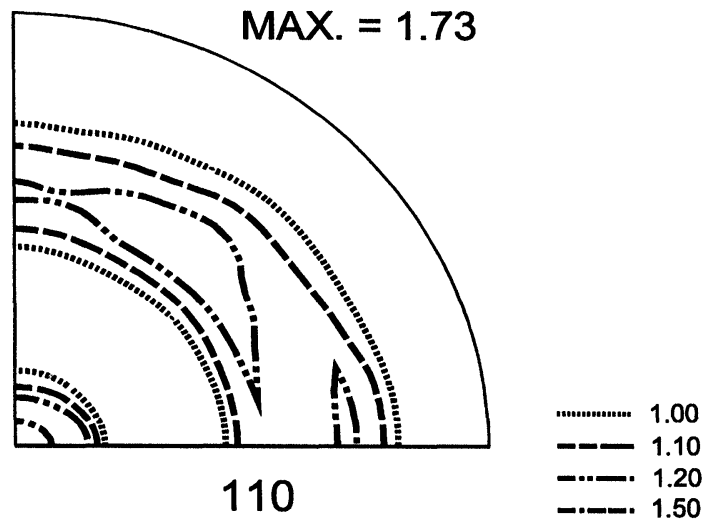

FIGURE 4 Pole figure of the cold extruded steel sample. 
Figure 5 shows the distribution of the axial residual stress component vs. sample diameter of a full forward extruded specimen. Neutron diffraction and synchrotron diffraction reveal in very good agreement, that in the inner part of the specimen the residual stresses in radial direction $\sigma_{\mathrm{rr}}$, hoop $\sigma_{\phi \phi}$, and axial direction $\sigma_{\mathrm{zz}}$, are compressive. These compressive residual stresses are balanced by tensile residual stresses in the outer part of the sample (Modlen et al., 1992). At the surface of the sample again compressive stresses in hoop and axial direction were obtained by $\mathrm{X}$-ray diffraction. This residual stress distribution, which is characteristic for hollow as well as full forward extruded samples, can be linked to the deformation process during the cold forward extrusion (Tekkaya, 1986). Due to deformation obstruction at the shoulder of the die, the material flow at the outer surface is slower and more inhomogeneous than in the inner part of the specimen. Therefore, within the inner part of the samples the grains are homogeneously stretched whereas at the outer surface of the samples the grains are first compressed and stretched later, while passing the transient radius of the die. Thus, tensile residual stresses remain in the outer part, while the inner part of the samples is under compressive residual stresses. The same

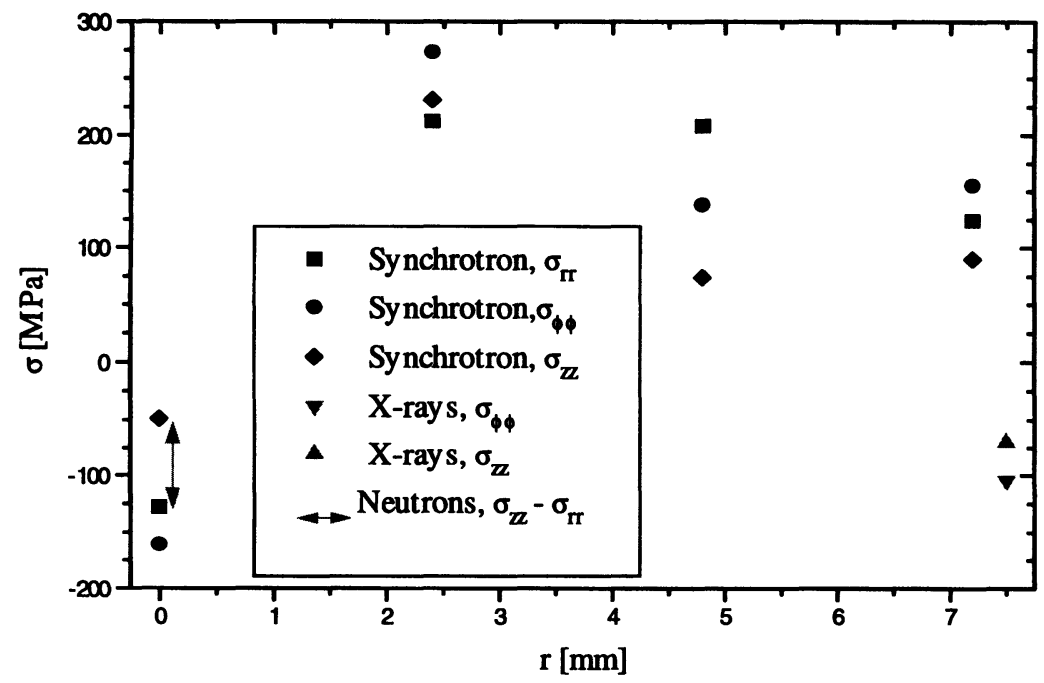

FIGURE 5 Residual stress distribution, full forward extruded sample, $\varphi=1.2$, steel grade C15. ( $\sigma_{r r}=\sigma_{\varphi \varphi}$ at $r=0$ hints to a small experimental error) 


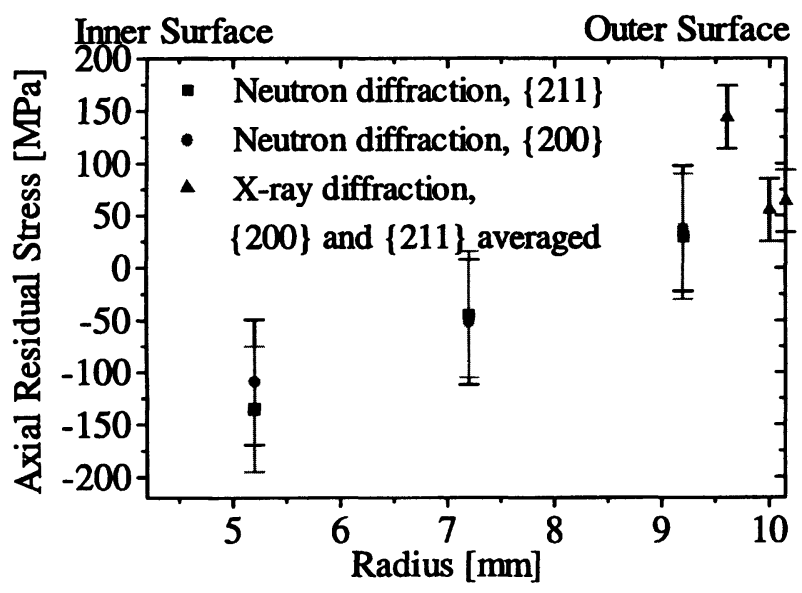

FIGURE 6 Axial residual stress vs. radius, hollow extruded specimen, C15, $\varphi=0.5$, ejected.

principle of residual stress formation holds for the hollow extruded samples. Therefore, the residual stress distributions obtained versus the diameter of a hollow sample (Fig. 6) is quite similar to the one of a full forward extruded sample.

Due to the weak texture and weak plastic anisotropy, which can be attributed to the low degree of natural strain of 0.5 , the axial stress component obtained for the two reflections 200 and 211 are in good agreement. Furthermore, the experimental results are in good qualitative agreement with Finite-Element-Calculations (Tekkaya, 1986) of the axial residual stress component (Fig. 7) in a sample that is slightly thicker but extruded with the same degree of natural strain.

\section{Hot Extrusion}

Whereas the steel $\mathrm{C} 15$ here can be regarded as a single-phase material, the alloy AlSi25Cu4Mgl is a multi-phase material containing large Si particles and smaller particles of an intermetallic phase (Fig. 8). Due to the low amount of intermetallic phase, it is not visible in the diffraction diagrams.

The texture of the hot extruded AlSi25Cu4Mgl alloy is a two-folded fibre texture with orientations $\langle 100\rangle$ and $\langle 111\rangle$ as fibre axis (Fig. 9), 


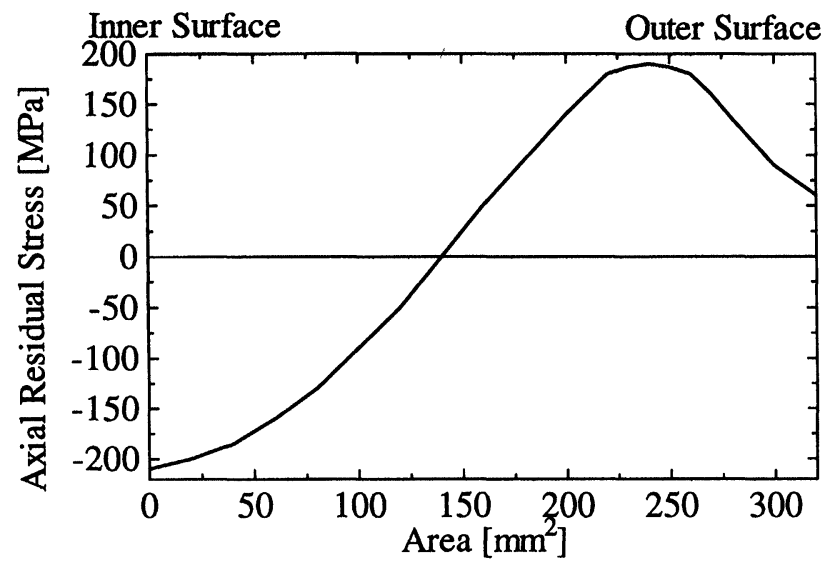

FIGURE 7 FEM-calculation (Tekkaya, 1986) of the axial residual stress vs. sample diameter, $\mathrm{C} 15, \varphi=0.5$, ejected.

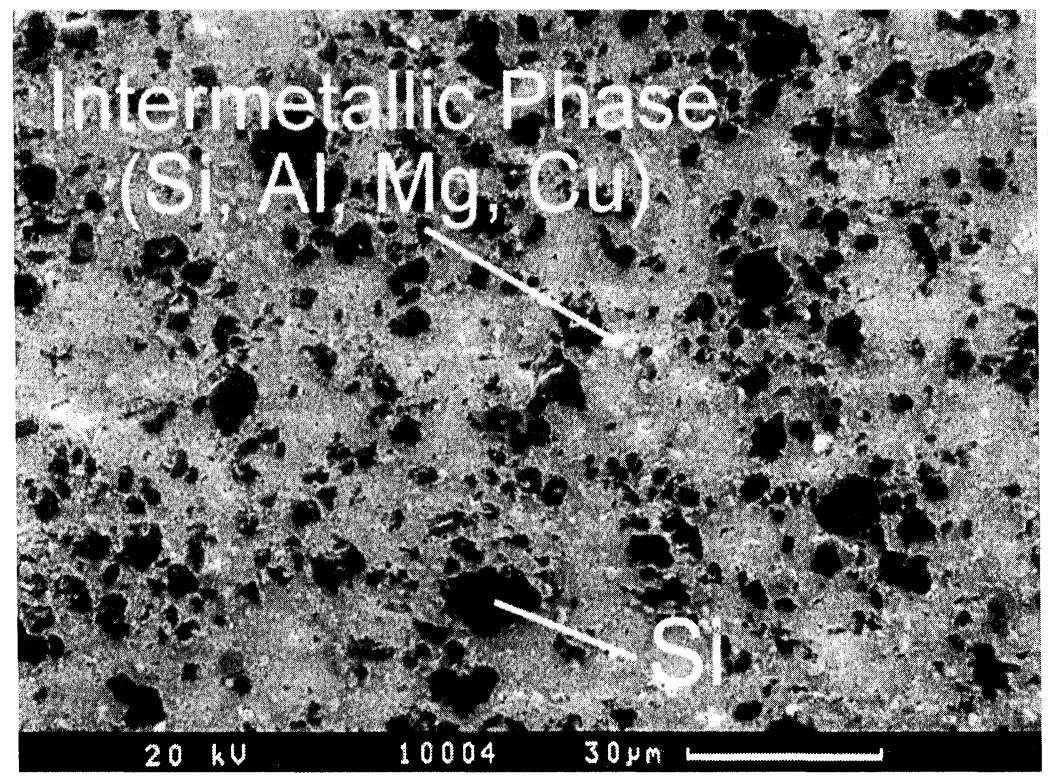

FIGURE 8 Microstructure of AlSi25Cu4Mg1. 

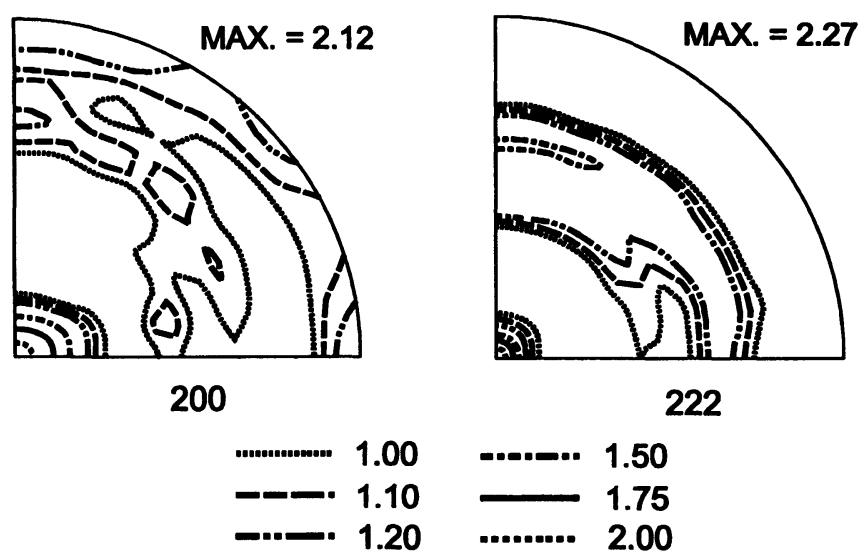

FIGURE 9 Pole figures of the hot extruded AlSi25Cu4Mg1 tube.

which is typical for extruded fcc materials (Wassermann and Grewen, 1962). The comparatively weak texture with regard to the degree of natural strain of the sample, according to Wassermann and Grewen (1962), can be attributed to the presence of the hard Si particles.

In opposition to the residual stress distribution in the cold extruded full and hollow samples the macroscopic residual stresses in axial direction are tensile within the inner part of the hot extruded sample, while the outer part of the hot extruded sample contains the balancing compressive residual stresses (Fig. 10).

The difference in the residual stress distribution vs. sample diameter on the one hand can be attributed to the larger degree of natural strain of $\varphi=2.8$ possible in hot extrusion, since tensile residual stresses also were observed in case of a cold extruded sample with a comparatively high degree of natural strain of $\varphi=1.6$ (Pyzalla et al., 1996; Pyzalla and Reimers, 1997). On the other hand, due to the macroscopic temperature gradient during cooling from the extrusion temperature, also compressive residual stresses arise near the outer surface of the tubes while balancing tensile residual stresses are induced at smaller sample diameters. Since the Al-alloy matrix of $\mathrm{AlSi} 25 \mathrm{Cu} 4 \mathrm{Mg} 1$ has a substantially larger thermal expansion coefficient than the Si particles, the cooling also produces phase specific residual microstresses. These are 


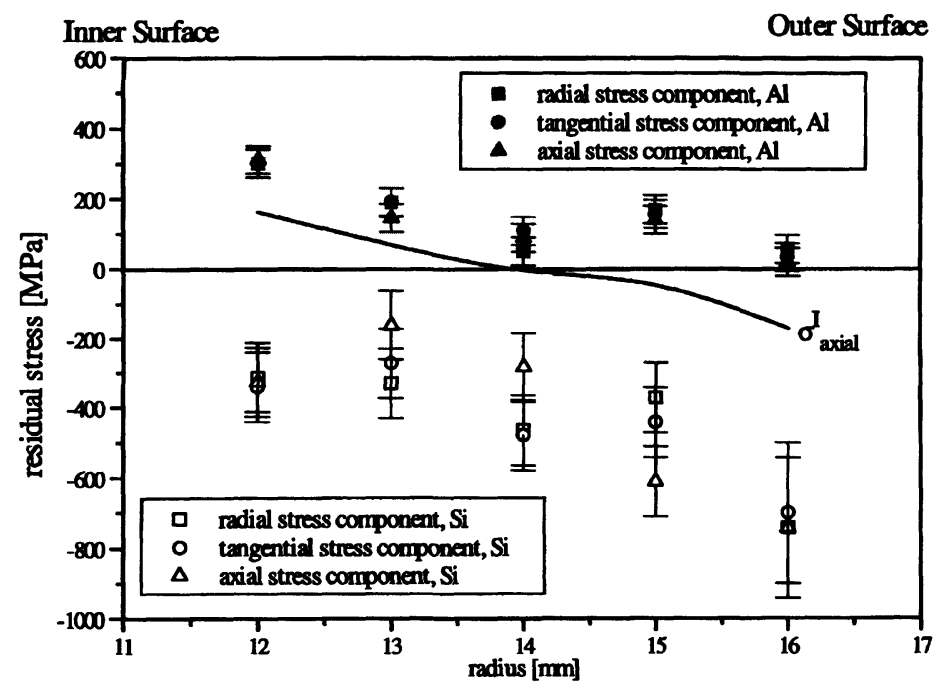

FIGURE 10 Residual stress distribution, full forward extruded sample, $\varphi=1.2$, steel grade C15.

compressive in the Si particles, while the Al-alloy matrix is under tensile residual microstresses.

\section{CONCLUSIONS}

The microstructure, the texture and the residual stress state of cold forward extruded and hot forward extruded samples were investigated by $\mathrm{X}$-ray, high energy synchrotron and neutron diffraction. The experiments reveal characteristic residual stress distributions vs. sample diameter. In case of the cold forward extruded samples compressive residual macrostresses are present in the rod kernel which are balanced by tensile residual stresses at larger sample diameters. In opposition, the hot extruded sample contains tensile residual stresses in the inner and compressive residual stresses in the outer part. From these experimental results it can be concluded that the residual stress distribution over the sample diameter can be largely influenced by the degree of natural strain and the extrusion temperature and, that thus a favourable residual stress state may be established using optimised process parameters. 


\section{Acknowledgements}

The authors would like to thank Prof. Dr.-Ing. K. Pöhlandt, Institute for Metal Forming, University of Stuttgart, Germany and Dr.-Ing. K. B. Müller, Technical University, Berlin, Germany, for manufacturing the samples and Dr. K. Liss of the ESRF Grenoble, France, for helping with the synchrotron experiments and the Deutsche Forschungsgemeinschaft (DFG) for financial support of this research project.

\section{References}

Frisch, J. and Thomsen, E.G. (1957). Trans. ASME, 79, 155.

Genzel, C., Reimers, W., Malek, R. and Pöhlandt, K. (1996). Mat. Sci. Eng. A, 205, 79. Hauk, V., Nikolin, H.-J. and Pintschovius, L. (1990). Z. Metallkd., 81, 556.

Hauk, V. and Sesemann, H. (1976). Z. Metallkd., 67, 646.

Hauk, V. and Vaessen, G. (1985). Z. Metallkd., 76, 102.

Macherauch, E. and Müller, P. (1961). Z. Angew. Phys., 13, 305.

Modlen, G.F., Webster, P.J., Wang, X. and Mills, G. (1992). Conf. Sheet Metal, p. 171, Birmingham.

Moore, M.G. and Evans, W.P. (1958). SAE Trans., 66, 340.

Pyzalla, A., Genzel, C., Reimers, W. and Pöhlandt, K. (1996). Metall., 50, 787.

Pyzalla, A. and Reimers, W. (1997). Residual stresses and texture in cold forward extrusion. In Competitive Advantages by Near-Net-Shape Manufacturing, edited by H.-D. Kunze, p. 175. Oberursel: DGM-Informationsgesellschaft Verlag.

Tekkaya, A.E. (1986). Ermittlung von Eigenspannungen in der Kaltmassivumformung. Universität Stuttgart, Thesis.

Wassermann, G. and Grewen, J. (1962). Texturen metallischer Werkstoffe, Berlin: Springer. 